\title{
METODOLOGÍAS PARA DETERMINAR LA RETENCIÓN DE HUMEDAD Y LA DENSIDAD EN EL COMPOST
}

\section{METHODOLOGIES TO DETERMINE THE WATER HOLDING CAPACITY AND DENSITY IN COMPOST}

\author{
${ }^{1}$ Tello P, Lily D. y ${ }^{2}$ Vega R, Ruby A.
}

\begin{abstract}
Resumen
Dos metodologías, TMECC (Test Methods for the Examination of Composting and Compost) y LASPAF (laboratorio de análisis de suelos y plantas de la UNALM), fueron utilizadas para determinar la capacidad de retención de agua y la densidad en compost con el objetivo de determinar si tienen correlación. Para ello, se realizaron los análisis estadísticos usando el modelo de regresión a través del origen en dos variables físicas del compost, la capacidad de retención de agua y la densidad, en 15 muestras. En la capacidad de retención de agua se obtuvo que los resultados del LASPAF seguían la tendencia de los resultados de la TMECC; sin embargo, para una mayor aproximación debemos multiplicar los valores del LASPAF por el factor de corrección de 0.46351. En densidad, los resultados fueron muy similares no sólo en tendencia, sino que el factor de corrección fue muy cercano a uno (0.90136).
\end{abstract}

Palabras claves: capacidad de retención de humedad, compost, densidad, metodología, metodología TMECC.

\begin{abstract}
Two methodologies, TMECC (Test Methods for the Examination of Composting and Compost) and LASPAF (laboratory analysis of soil and plants UNALM), were used to determine the water holding capacity and density in compost with the aim of determine any correlation. For this, statistical analysis using regression through the origin in physical variables of the compost was done on 15 samples, the water holding capacity and density. The water holding capacity and the results obtained from LASPAF followed the trend of the results TMECC; however, for a closer result we must multiply values by LASPAF by a correction factor, 0.46351 . Density, the results were very similar not only in trend even the correction factor was very close to one (0.90136).
\end{abstract}

Key words: compost, density, methodology, TMECC methodology, water holding capacity.

\section{Introducción}

En los últimos años, el interés de estimar la calidad del compost se ha incrementado, ya que constituye un beneficio para la agricultura y el medio ambiente. El compost debe ser un sustrato amigable para la planta, el suelo y el ambiente, no debe presentar contaminantes orgánicos o inorgánicos; a su vez, su elaboración, conservación y transporte debe ir de la mano con la responsabilidad social para no ocasionar problemas a la salud (Baffi et al., 2007). Por ello, se han adaptado metodologías no específicas para la evaluación de las materias orgánicas; asimismo, la heterogeneidad de los procedimientos para conocer las características y/o propiedades del compost promovió la aparición de diversos estándares que permiten determinar su calidad basados en las normativas e intereses de cada organización y/o país; por ello, es usual encontrar diferentes resultados de una misma muestra, lo que genera una gran confusión en la interpretación y disconformidad en relación a la aplicación de la prueba.
Al ser un sustrato rico en microorganismo, el enfoque de su evaluación hace más importante las mediciones de su estabilidad biológica a través de su actividad microbial; sin embargo, lo tedioso, el costo y el tiempo de las determinaciones biológicas hacen más atractivas a las determinaciones físicas y químicas, las cuales suelen ser más económicos y usar menos tiempo, sobre todo las mediciones químicas (Dell'Abate et al., 1998; Tittarelli et al., 2002); es por ello, que en la actualidad el gran universo de normativas se ha orientado por categorizar al compost en función de sus características químicas. Varios autores (Inbar et al., 1990; Barberis and Nappi, 1996; Chen et al., 1996; Itävaara et al., 2002) están de acuerdo que las metodologías o técnicas aisladas no logran ser exitosas para evaluar calidad en compost, debido al gran espectro de materias primas con los que se elaboran; por ejemplo, la diversidad de estiércoles usados (vacuno lechero, vacuno de carne, animales menores, gallinaza, etc.) afecta significativamente la estabilidad biológica del compost (Mondini et al., 2003). Debido a ello es que se genera la controversia y muchas interrogantes, incluso en

${ }^{1}$ Universidad Nacional Agraria La Molina. Lima, Perú. E-mail: ampelio@lamolina.edu.pe, ltello@lamolina.edu.pe

${ }^{2}$ Universidad Nacional Agraria La Molina. Lima, Perú. E-mail: rvega@lamolina.edu.pe 
una misma investigación, sobre si se requiere o no más de un método para determinar la estabilidad biológica de un compost. La técnica del respirómetro resulta ser una medida satisfactoria de la estabilidad biológica del compost por lo que con el tiempo se ha vuelto un proceso de rutina para determinar calidad del compost en diversos países como Italia, Alemania, Austria, Reino Unido, Estados Unidos y actualmente son métodos codificados internacionalmente (ASTM, 1996; The U.S.Composting Council, 1997). Por otro lado, también se acepta el uso integrado de diferentes índices de medición que describen las propiedades de los materiales usados para obtener compost, lo cual brinda mayor información de la composición del compost y podría servir para un uso más apropiado del mismo (Baffi et al., 2007).

Es claro, que cada método puede tener limitantes únicas que podrían afectar el resultado; por ello, la TMECC (Test Methods for the Examination of Composting and Compost) estableció una normativa que unifica las metodologías específicas para determinar las propiedades del compost, integrando las evaluaciones empleadas en diversos laboratorios y recomendando los pasos a seguir para analizar materias orgánicas (Thompson et al., 2001).

La adición de materia orgánica provee de nutrientes a las plantas y de beneficios asociados al mejoramiento de las propiedades físicas y químicas del suelo (Carter et al., 2003; Christensen and Johnston, 1997). La búsqueda de la ampliación de fronteras agrícolas en suelos de fertilidad pobre motivó al hombre a mejorar las prácticas agrícolas que le permitan obtener mayor cantidad de sustratos como el compost. El compost se define como la materia orgánica estabilizada después de una reacción bio-oxidativa controlada (Zucconi, 1986) formando parte del proceso de humificación (Leege y Thompson, 1997).

Las propiedades del compost que toman interés para mejorar las características físicas del suelo son la capacidad de retención de agua, definida como la cantidad de agua retenida en los poros luego de la pérdida de agua gravitacional en un determinado tiempo y la densidad aparente es considerada como el peso por unidad de volumen (Shyamala, 2012). Sin embargo, los estudios se han orientado a calificar la calidad del compost en base a sus características químicas, puesto que las variables son rápidamente medibles gracias a equipos muy precisos y en especial por el interés de su contenido químico sobre el impacto ambiental (Fauci, et al., 1999; Ahmed, et al., 2007). Dado este contexto y teniendo en cuenta que el impacto de las características físicas de este abono orgánico genera en las propiedades del suelo (Eyras, et al., 1998; Mohee y Mudhoo, 2005), la presente investigación buscó comparar las metodologías para determinar capacidad de retención de agua y densidad propuestas por el TMECC y las aplicadas en el Laboratorio de Análisis de Suelos, Plantas, Aguas y fertilizantes (LASPAF) y así establecer su relación.

\section{Materiales y métodos}

\section{Ubicación del experimento}

El experimento se realizó en el Laboratorio de Manejo de Suelos y en el Laboratorio de Análisis de Suelos, Plantas, Aguas y Fertilizantes (LASPAF), dependencias del Departamento Académico de Suelos de la Facultad de Agronomía de la Universidad Nacional Agraria La Molina (UNALM).

\section{Características de los compost}

Los compost utilizados se encontraban en su fase de madurez y aún en pilas. Para determinar las evaluaciones se utilizó el material de 15 pilas de compost ubicadas en las instalaciones de la UNALM.

Cuadro 1. Características físico-químicas de los compost evaluados y las materias primas usadas para su elaboración.

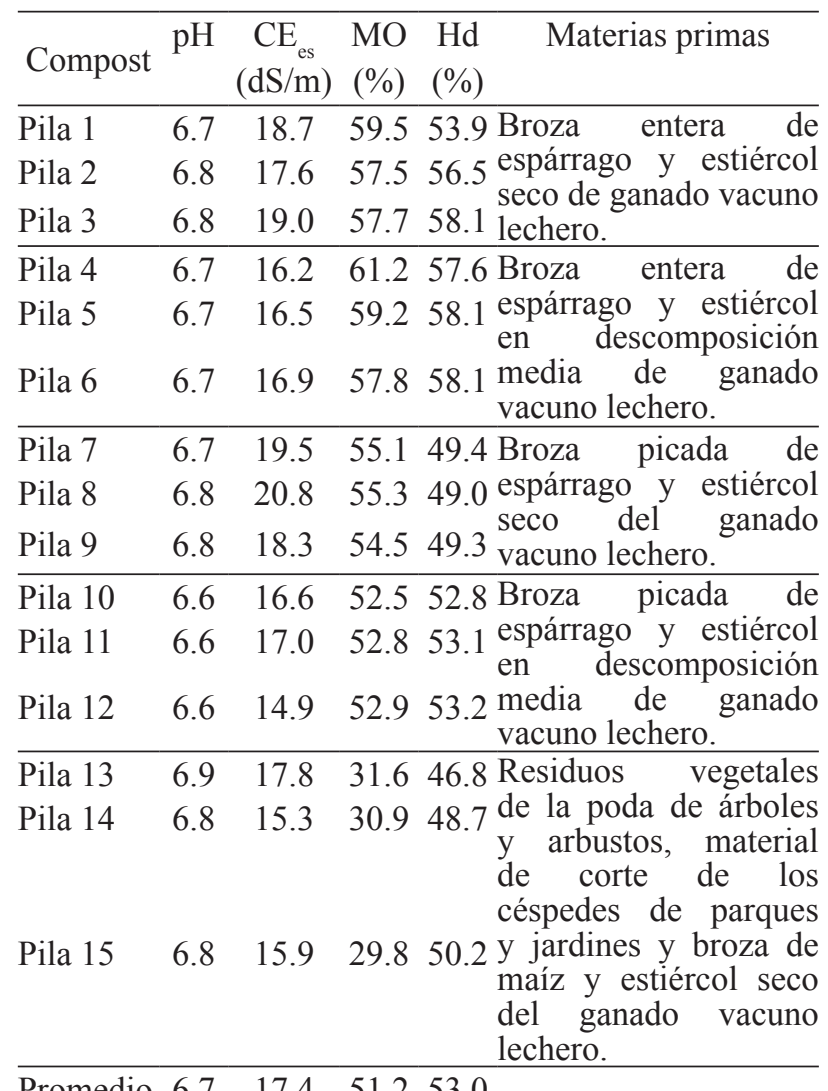

\begin{tabular}{lllll}
\hline Promedio & 6.7 & 17.4 & 51.2 & 53.0 \\
MAX & 6.9 & 20.8 & 61.2 & 58.1 \\
MIN & 6.6 & 14.9 & 29.8 & 46.8 \\
\hline
\end{tabular}

En el Cuadro 1 se puede apreciar que la característica química más resaltante de los compost utilizados es su contenido de materia orgánica. En las doce primeras pilas se observa niveles de materia orgánica por encima del $50 \%$, lo que va de la mano con la fuente de materia prima, broza de espárrago, un material con alto contenido de lignina y de difícil descomposición, por lo que el sustrato está compuesto de estructuras químicas más complejas 
y posee un mayor cantidad de carbono potencialmente oxidable. Asimismo, es importante enfatizar que los compost elaborados con broza de espárrago picada sólo favorecieron la producción del sustrato en un menor tiempo; mientras que aquellos compost elaborados con estiércol de descomposición media, el cual es producto del humedecimiento del estiércol seco y su compostación durante tres semanas, presentaban partículas más pequeñas. Por otro lado, los compost producidos con los diversos materiales vegetales de parques y jardines, a pesar de poseer fragmentos groseros de los leños, fueron más consistentes.

Las muestras de compost utilizadas para el estudio fueron cernidas a través de un tamiz de $3 / 8$ pulgadas, como indica la norma TMECC para su homogenización. De cada pila de compost maduro, tamizado y homogenizado se obtuvo una muestra compuesta de tres sub-muestras tomadas en la pila: sub-muestra del tercio superior, tercio medio y tercio inferior de la dirección del eje central.

\section{Análisis físicos \\ Prueba rápida para determinar capacidad de retención de agua (CRA) y densidad en compost según TMECC}

Determinación del peso de la muestra seca a estufa: Tarar la cubeta de precipitación (beaker) de $150 \mathrm{~mL}$ usando la balanza analítica, añadir $50 \mathrm{cc}$ de muestra de compost y pesar $\left(\mathrm{A}_{50}\right)$. Secar la muestra a estufa $70 \pm 5^{\circ} \mathrm{C}$ de 18 a 24 $\mathrm{h}$ y anotar peso seco $\left(\mathrm{O}_{50}\right)$.

Modificación de jarras graduadas de polipropileno de $2000 \mathrm{~mL}$ : Hacer agujeros espaciados uniformemente de $3 \mathrm{~mm}$ en la parte inferior de las jarras graduadas de 2000 $\mathrm{mL}$ y sellarlos con masking tape temporalmente para prevenir fugas. Pesar la jarra modificada (A).

Llenado de las jarras graduadas modificadas de $2000 \mathrm{~mL}$ con compost: Transferir $600 \mathrm{cc}$ de compost con ayuda del embudo. Asegurar la distribución uniforme de la muestra en la jarra dejándola caer libremente desde 15 $\mathrm{cm}$ de altura sobre una colchoneta de polietileno. Repetir la adición de 600 cc de compost y la acción de dejar caer libremente 2 veces más el material contenido (3 veces en total). Si después de la tercera caída libre, el material está por debajo de $1800 \mathrm{~mL}$ añadir compost hasta enrazar $1800 \mathrm{~mL}$ pero no más de $3 \mathrm{~cm}$.

Determinación del peso de la muestra (C): Pesar la jarra graduada de $2000 \mathrm{~mL}$ con los $1800 \mathrm{~mL}$ de compost (B) y restar el peso inicial de la jarra graduada modificada (A).

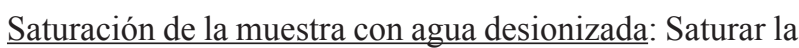
muestra de compost con $1000 \mathrm{~mL}$ de agua desionizada, verter lenta y cuidadosamente el agua hasta que la parte superior del compost se defina un espejo de agua (lámina de agua), teniendo cuidado de no añadir agua en exceso. Cubrir la jarra modificada de $2000 \mathrm{~mL}$ usando parafilm por 5 minutos y repetir la adición de agua en períodos de descanso de 5 minutos chequeando la lámina de agua. La saturación finaliza cuando el compost no absorba más agua y en la parte superior del compost se observe el espejo. Anotar la cantidad de agua usada para la saturación y guardar el agua no usada.

Estimación del espacio poroso: Colocar la jarra modificada sobre una rejilla de $3.8-5 \mathrm{~cm}$ de diámetro y encima de otra jarra de $2000 \mathrm{~mL}$. Retirar el masking tape y capturar lo drenado para reuso. Después de 30 minutos cubrir hoyos de drenaje con masking tape y nuevamente saturar lenta y cuidadosamente la muestra de compost con el agua drenada. Repetir 3 veces para asegurar que el total humedecimiento; si es necesario añadir más agua de la capturada. Pesar las jarras de $2000 \mathrm{~mL}$ con el compost saturado (D) y restarle el peso del compost (C) para obtener el peso del agua no drenada (E) (W1800 ws, no drain). Leer y anotar el volumen de compost saturado (F) (V $1800 \mathrm{ws,} \mathrm{no} \mathrm{drain).} \mathrm{Verificar} \mathrm{el} \mathrm{volumen} \mathrm{de} \mathrm{agua} \mathrm{total}$ añadido por comparación de diferencias de pesos entre el peso de la jarra graduada de $2000 \mathrm{~mL}$ con los $1800 \mathrm{~mL}$ de compost (B) y el peso de la jarra graduada de 2000 $\mathrm{mL}$ con el compost saturado (D). Dejar libres los agujeros y recibir el agua drenada del compost durante 4 horas, cubriendo la jarra para minimizar la pérdida de agua por evaporación. Leer y anotar el volumen de agua liberada del compost en las 4 horas (G). Determinar el peso del agua saturada después de 4 horas de drenaje (I) pesando la jarra graduada de $2000 \mathrm{~mL}$ que contiene el compost saturado luego de 4 horas $(\mathrm{H})$ y restar el peso de la jarra de $2000 \mathrm{~mL}$ modificada (A) (W1800 ws, after drain). Comparar y verificar la determinación del volumen de espacio aéreo dividiendo el volumen de agua drenada con el total de volumen de agua añadida para saturar la muestra de compost.

Volumen de agua saturada: Leer y anotar el volumen de agua saturada del compost húmedo en el beaker graduado ( $\pm 5 \mathrm{~mL}$ ) que equivalente a la máxima capacidad de retención de agua.

Fórmula para determinar Densidad (dens) según TMECC Dens $=(\mathrm{C}(\mathrm{O} 50 / \mathrm{A} 50)) / 1800$

Método para determinar la retención de humedad (rhd) según el Laboratorio de Análisis de Suelos, Plantas, Aguas y Fertilizantes (LASPAF)

La metodología usada es una adaptación de la metodología utilizada por Mbah y Odili (1998). Consiste en construir un sistema de drenaje con embudos con la capacidad de contener el volumen de material a usar, manguera y llave. Colocar un papel de filtro sobre el embudo y humedecerlo ligeramente. Cerrar la llave del sistema para evitar el drenaje. Pesar aproximadamente $500 \mathrm{~g}$ de compost mullido y colocarlo en el embudo. Agregar agua destilada en exceso y en cantidad suficiente hasta producir un sobrenadante. Dejar el material saturado para asegurar que se hidrate durante una semana. En caso de observar que pierde la condición de sobrenadante, agregar más agua. Cuando se observe que el material orgánico ya no se hidrate más y se mantenga en volumen constante, abrir la 
llave y dejar drenar durante 24 horas. Tomar una porción del compost drenado para determinar su contenido de humedad, el cual corresponde a su retención de agua. Colocar el material en una lata de aluminio. Pesar la porción de compost tomada (Wh). Secar la porción de compost tomada en la estufa a temperatura no mayor de $80{ }^{\circ} \mathrm{C}$. Pesar la porción secada a estufa (Ws).

Cálculos: Humedad $(\%)=(\mathrm{Wh}-\mathrm{Ws}) / \mathrm{Ws})^{*} 100$, donde Wh: Peso de la muestra húmeda y Ws: Peso de la muestra seca.

Método para determinar Densidad (dens) según el Laboratorio de análisis de suelos, plantas, aguas y fertilizantes (LASPAF)

Esta metodología se aplica en el compost drenado obtenido en la determinación de la máxima retención de humedad referida en la metodología anterior. Construir cilindros de 30 ó 40 centímetros cúbicos de capacidad $(\mathrm{Vc})$, con mayor área superficial que altura. Introducir cuidadosamente el cilindro dentro del compost drenado que está depositado en el embudo. Se recomienda no disturbar el material y considerar la resistencia que puede ofrecer el material. Retirar el cilindro con ayuda de una espátula o cuchillo. Enrazar los bordes del cilindro con una espátula o cuchillo. Depositar todo el compost contenido en el cilindro en una lata de aluminio. Secar el material en la estufa a temperatura no mayor de $80^{\circ} \mathrm{C}$. Pesar el material secado en la estufa (Ws).

Cálculos: Densidad $\left(\mathrm{g} / \mathrm{cm}^{3}\right)=$ Ws/Vc, donde Ws: Peso de la muestra seca y Vc: Volumen del cilindro.

\section{Análisis estadísticos}

Con ayuda del paquete estadístico $\mathrm{R}$, se realizaron los análisis estadísticos para las variables físicas en estudio basándonos en los modelos alométricos, con el propósito de establecer la variación de los métodos del LASPAF con respecto a los métodos establecidos por el TMECC y encontrar el factor de corrección que aproxime ambos resultados. Se usó el análisis de regresión a través del origen, puesto que la metodología ejecutada comprendió la aplicación de un modelo de medición (uso de instrumentos de medición) que no considera intercepto y sólo establece corrección entre dos variables. Por tal motivo, no se trabajó el análisis de regresión lineal simple, que considera intercepto e involucra una traslación de la relación lineal (movimiento del instrumento) y una valor de ponderación entre las dos variables en estudio.

El modelo de regresión a través del origen es:

$\mathrm{Y}=\boldsymbol{\beta}_{1} \mathrm{X}+\varepsilon$

Donde:

$\mathrm{Y}=$ Variable dependiente

$\beta 1=$ Constante

$\mathrm{X}=$ Variable independiente

$\varepsilon=$ Error
Asimismo, para validar el instrumento de análisis se realizó dos análisis de correlación para conocer la exactitud en función al valor (acercamiento exacto de un valor al otro) y la precisión en función a la variación, correlación de Pearson y Spearman, respectivamente. Ambos deben presentar valores de altos valores de correlación para ser validados (Montgomery y Runger, 1998).

\section{Resultados y discusión}

El análisis de regresión a través del origen cumple los supuestos exigidos. En el plano cartesiano los valores de las variables tienden a una línea recta. Los Residuals vs. Fitted muestran que todos los valores se acercan a cero y los Residuals vs. Leverage exhiben a los valores extremos lejos de la línea de referencia; además, los datos tienen un comportamiento normal. Debido a ello las variables en estudio rhd y dens muestran la siguiente tendencia:

\section{$\underline{\text { Retención de humedad }}$}

El Cuadro No2 muestra valores entre $190.270 \%$ y $240.750 \%$ para la rhd bajo la metodología LASPAF y con la metodología TMECC encontramos rangos de $85.100 \%$ a $125.900 \%$. Los resultados hallados en ambas metodologías muestran marcadas diferencias; pero con una similar tendencia en cuanto a los compost elaborados con estiércol en descomposición media, los cuales presentan las mayores retenciones de humedad por poseer mayor cantidad de partículas más pequeñas. En promedio se puede decir que con el método LASPAF los compost pueden retener el $215.170 \%$ de agua con respecto a $100 \mathrm{~g}$ de peso seco del material; mientras, por la metodología TMECC se han determinado valores más bajos, los compost en promedio retienen el $105.847 \%$ de agua con respecto a $100 \mathrm{~g}$ de peso seco del material.

Cuadro 2. Retención de humedad determinados por los métodos LASPAF y TMECC.

\begin{tabular}{lcc}
\hline \multicolumn{1}{c}{ Compost } & rhdLASPAF & $\begin{array}{c}\text { rhdTMECC } \\
(\%)\end{array}$ \\
\hline Pila 1 & 195.910 & 100.900 \\
Pila 2 & 190.270 & 119.300 \\
Pila 3 & 209.310 & 86.900 \\
Pila 4 & 226.950 & 109.900 \\
Pila 5 & 240.750 & 101.500 \\
Pila 6 & 232.210 & 108.600 \\
Pila 7 & 194.020 & 125.900 \\
Pila 8 & 207.730 & 120.300 \\
Pila 9 & 204.880 & 109.100 \\
Pila 10 & 228.200 & 112.000 \\
Pila 11 & 230.290 & 115.100 \\
Pila 12 & 225.590 & 107.000 \\
Pila 13 & 220.660 & 90.800 \\
Pila 14 & 216.890 & 95.300 \\
Pila 15 & 203.890 & 85.100 \\
\hline
\end{tabular}


Continuación de Cuadro 2

\begin{tabular}{lcc}
\hline PROMEDIO & 215.170 & 105.847 \\
MAX & 240.750 & 125.900 \\
MIN & 190.270 & 85.100 \\
\hline
\end{tabular}

Hay suficiente evidencia estadística $(P<0.001)$ para establecer que existe una línea que relaciona la metodología TMECC y LASPAF; dado que el aporte de la variable rhd a la relación es altamente significativo y puede ser considerada dentro del modelo de regresión lineal sin intercepto.

Según la regresión sin intercepto determinada, el factor de corrección 0.48904 multiplicado a los valores de rhdLASPAF permite corregir los resultados obtenidos y los aproxima a los valores que se obtendrían al determinarse por la metodología del TMECC (Fig. 1), estableciéndose una relación directamente proporcional. Asimismo, es resaltante que el $98.08 \%$ de los resultados obtenidos en rhdTMECC son explicados por rhdLASPAF.

En el análisis de correlación de Pearson no se encuentra evidencia estadística para establecer un acercamiento entre los valores hallados en ambos métodos. En la correlación de Spearman no hay diferencia significativa entre los valores correlacionados, por lo que no hay asociación de rango.

Bajo las condiciones del trabajo experimental se ha encontrado que las metodologías desarrolladas son aplicables y adaptables a la diversidad de materiales compostados $(\mathrm{CV}=14.45)$. Las características de la población evaluada han permitido que el espectro de resultados sea amplio, puesto que la variedad de fuentes de materias primas produjeron compost con heterogéneos e irregulares tamaños de partículas, incluso de tamaños muy variables después de tamizarlos en un tamiz de una pulgada. Es el tamaño de partícula lo que mayor influencia ha ejercido en la capacidad de retener humedad en los compost, puesto que con un menor tamaño de partícula se tiene una mayor área de superficie específica que incrementa la adsorción de agua (Brady y Weil, 2008; Mbah y Odili, 1998).

El $\mathrm{R}^{2}$ de 0.9808 nos indica que ambas metodologías siguen semejante tendencia al presentar líneas paralelas; es decir, para tener valores equivalentes en base a la metodología TMECC, los resultados obtenidos en la variable rhdLASPAF habría que multiplicarlos por el factor de corrección 0.48904. Este bajo valor del factor hallado refleja las variaciones en los procedimientos seguidos para la determinación de la rhd en ambas metodologías.

Si comparamos los procedimientos de ambas metodologías para determinar rhd, se observa que la del TMECC es más tediosa, cuidadosa y toma más tiempo; mientras que la metodología del LASPAF es más práctica y rápida. Bajo este contexto, el factor de corrección permite trabajar con la metodología más práctica (LASPAF) y ajustarla para obtener valores determinados por una metodología normada exclusivamente para compost (TMECC).

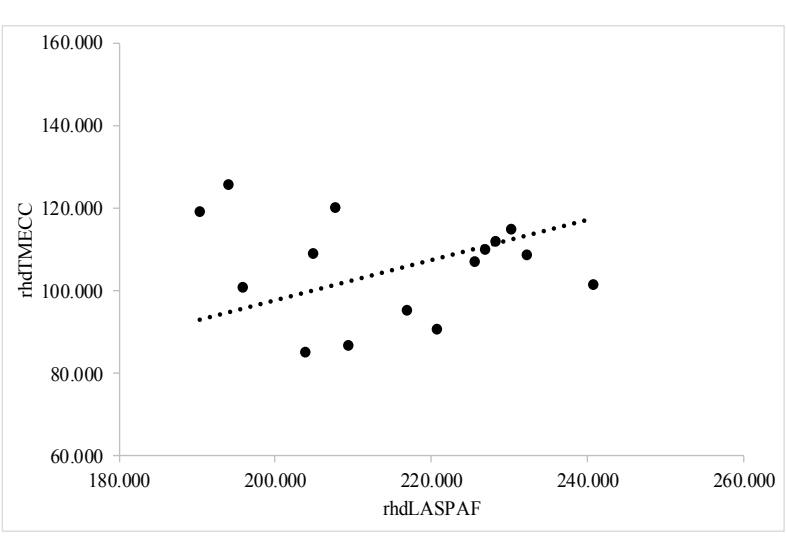

Figura 1. Diagrama de dispersión de la relación entre la rhd determinada por la metodología TMECC y LASPAF para diversas muestras de compost.

\section{Densidad}

En el Cuadro 3 encontramos un amplio rango, de 0.220 g.cm a 0.432 g.cm cm $^{-3}$, para las densidades determinadas bajo la metodología LASPAF y un rango más corto, de 0.190 g.cm $\mathrm{cm}^{-3}$ a $0.340 \mathrm{~g} . \mathrm{cm}^{-3}$, para los resultados hallados con la metodología TMECC. Los resultados hallados en ambas metodologías no muestran marcadas diferencias; pero si es evidente que los compost elaborados con los diversos materiales de los parques y jardines presentan los mayores valores de densidad por su mayor consistencia y variedad de materias primas; mientras que los elaborados con broza de espárrago exhiben los valores más bajos, puesto a que el material no se llega a descomponer totalmente y hay una menor reducción de volumen con respecto a las pilas 13 a la 15 . Los promedios indican que para el método LASPAF $0.274 \mathrm{~g}$ ocupa un centímetro cúbico y en la metodología TMECC $0.251 \mathrm{~g}$ de compost ocupa un centímetro cúbico, lo que exhibe que no hay una diferencia marcada entre los resultados obtenidos para la variable densidad.

Se ha encontrado evidencia estadística altamente significativa para establecer que existe una línea de regresión lineal sin intercepto entre las variables densTMECC y densLASPAF; puesto que la variable densidad explica la relación establecida $(P<0.001)$, por lo que puede ser considerada dentro del modelo estadístico. El factor de corrección encontrado, 0.92874 (Fig. 2), transforma los valores de densLASPAF a valores próximos reales que se obtendrían al determinarse por la metodología del TMECC, estableciéndose una relación directamente proporcional. Además, se puede indicar que la densTMECC se explica en un $97.43 \%$ por la variable densLASPAF.

En cuanto al análisis de correlación de Pearson, la evidencia estadística indica que el acercamiento de los valores registrados en las evaluaciones por ambos métodos es altamente significativo, hay representatividad punto a punto; lo que corrobora al observar resultados tan similares en el Cuadro 2. En la correlación de Spearman no hay diferencia significativa entre los valores 
correlacionados, por lo que no hay asociación de rango.

El $\mathrm{R}^{2}$ de 0.9743 reitera que ambas metodologías siguen semejante comportamiento; pero a diferencia de la evaluación de rhd las líneas paralelas son más cercanas; es por ello que encontramos un factor de corrección más alto, 0.92874. Al presentar el factor un valor muy próximo a uno, nos muestra que el principio de evaluación de ambos métodos sigue el mismo lineamiento y el error es menor.

Cuadro 3. Densidad de compost determinados por los métodos LASPAF y TMECC.

\begin{tabular}{lcc}
\hline \multicolumn{1}{c}{ Compost } & densLASPAF & $\begin{array}{c}\text { densTMECC } \\
\left(\mathrm{g} . \mathrm{cm}^{-3}\right)\end{array}$ \\
\hline Pila 1 & 0.260 & 0.270 \\
Pila 2 & 0.264 & 0.230 \\
Pila 3 & 0.253 & 0.280 \\
Pila 4 & 0.252 & 0.190 \\
Pila 5 & 0.269 & 0.230 \\
Pila 6 & 0.264 & 0.200 \\
Pila 7 & 0.252 & 0.270 \\
Pila 8 & 0.237 & 0.260 \\
Pila 9 & 0.257 & 0.270 \\
Pila 10 & 0.230 & 0.270 \\
Pila 11 & 0.270 \\
Pila 12 & 0.256 & 0.280 \\
Pila 13 & 0.220 & 0.330 \\
Pila 14 & 0.386 & 0.270 \\
Pila 15 & 0.334 & 0.340 \\
\hline Promedio & 0.432 & 0.264 \\
MAX & 0.278 & 0.340 \\
MIN & 0.432 & 0.190 \\
\hline
\end{tabular}

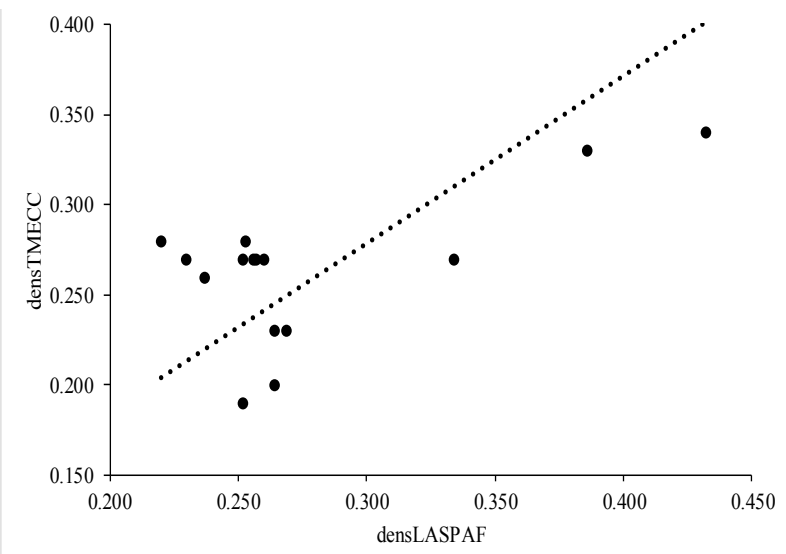

Figura 2. Diagrama de dispersión de la relación entre la densidad determinada por la metodología TMECC y LASPAF para diversas muestras de compost.

A diferencia de los procedimientos para rhd, las evaluaciones de densidad son sencillas, rápidas $\mathrm{y}$ semejantes.

\section{Conclusiones}

Ambas metodologías son adecuadas para determinar densidad aparente y retención de humedad en muestras de compost.

Para que los resultados de rhd por el método del LASPAF sean similares a los resultados del TMECC, deben ser multiplicados por el factor de corrección de 0.48904 .

Para que los resultados de densidad por el método del LASPAF representen más a los resultados del TMECC, deben ser multiplicados por el factor de corrección de 0.92874 .

\section{Literatura citada}

Ahmed, M.; Idris, A. and Syed, S. R. (2007). Physicochemical characterization of Compost of the industrial tannery sludge. Journal of Engineering Science and Technology Vol. 2 N 1 (2007) 81-94. School of Engineering, Taylots University College.

Baffi, C.; Dell, M.; Nassissi, A.; Silva, S.; Benedetti, A.; Genevini, P. y Adani, F. (2007). Determination of biological stability in compost: A comparison of methodologies. Soil Biology \& Biochemistry 39 (2007) 1284-1293.

Barberis, R. and Nappi, P. (1996). Evaluation of compost stability. In: De Bertoldi, M., Sequi, P., Lemmes, B., Papi, T. (Eds.). The Science of Composting. Blackie Academic and Professional, Glasgow, Scotland, pp. 175-184.

Blackshaw, R. E.; Molnar, L. J. and Larney, F. J. (2005). Fertilizer, manure and Compost effects and weed growth and competition with winter wheat in western Canada. Crop Protection 24 (2005) 971-980. Available online at www.sciencedirect.com

Brady, N.C. and Weil, N. C. (2008). The Nature and Properties of Soils. $14^{\text {th }}$ ed. Pearson Prentice Hall. 975pp.

Carter, M. R.; Sanderson, J. B.; and MacLeod, J. A. (2003). Influence of compost on the physical properties and organic matter fractions of a fine sandy loam throughout the cycle of a potato rotation. Canadian Journal of Soil Science.

Chen, Y.; Inbar, B.; Chefetz, B. and Hadar, Y., 1996. Compost and recycling of organic wastes. In: Rosen, D., Tal Or, E., Hadar, I., Chen, Y. (Eds.). Modern Agriculture and Environment. Kluwer Academic Publishers, Dordrecht, The Netherlands, pp. 341-362.

Christensen, B. T. and Johnston, A. E. (1997). Soil organic matter and soil quality. Lessons learned from long-term experiments at Askov and Rothamsted. Pages 399-430 in E.G. Gregorich and M. R., eds. Soil quality for crop production and ecosystem health. Elsevier, Amsterdam, The Netherlands.

Dell'Abate, M. T.; Canali, S.; Trinchera, A.; Benedetti, A. and Sequi, P., (1998). Thermal analysis in the evaluation of compost stability: a comparison with humification 
parameters. Nutrient Cycling Agroecosystems 51, 217224.

Erhart, E. and Hartl, W. (2010). Compost Use in Organic Farming. Chapter 11. Sustainable Agriculture Review 4, DOI 10.1007/978-90-481-8741-6-11, springer Science, Business Media B.V. 2010.

Eyras, M.C.; Rostagno, C.M. and Defossè, G.E.; (1998). Biological Evaluation of Seaweed Composting. Compost Science and Utilization, (1998), vol. 6, N 4, 74-81.

Fauci, M.F.; Bezdicek, D.F.; Caldwell, D. and Finch, R (1999). End Product Quality and Agronomic Performance of Compost. Compost Science and Utilization, (1999), vol. 7, N 2, 17-29.

Inbar, Y.; Chen, Y.; Hadar, Y. and Hoitink, H.A.J. (1990). New approaches to compost maturity. Biocycle 31, 64-69.

Itävaara, M.; Venelampi, O. and Kapanen, A. (2002). Compost maturity problems associated with testing. In: Insam, H., Riddech, N., Klammer, S (Eds.). Microbiology of Composting. Springer, Heidelberg, Germany, pp. 373382.

Leege, P. B. (1998). Introduction of Test Methods for the Examination of Composting and Compost. Beneficial CoUtilization of Agricultural, Municipal and Industrial byProducts. 269-282 pp.

Leege, P. and Thompson, W. (------)Test Methods for Examination Composting and Compost. The US Composting Council, 4424 Montgomery Avenue, Suite 102, Bethesda, Maryland 20814. USA.

Lopez, M; Huerta-Pujol, O; Martinez-Farre, X; Soliva, M. (2010). Approaching compost stability from Klason lignin modified method: Chemical Stability Degree for $\mathrm{OM}$ and $\mathrm{N}$ quality assessment. Resources, Conservation and Recycling, 55 (2010): 171-181.

Lynch, D.H; Voroney, R. P. and Warman, P. R. 2005. Soil Physical Properties and Organic Matter Fractions under Forages Receiving Composts, Manure or Fertilizer. Compost Science and Utilization, (2005), vol. 13, N 4, 252-261.

Mader, P.; Szakova, J. and Kucera, J. 1994. Interlaboratory Analysis of IRM NCS-21 Compost Vitahun. Biological Trace Element Research Editor: G.N.Scharauzer 1994 by Humana Press inc.

Mbah, B. and Odili, P. (1998). Changes in moisture retention properties of five waste materials during short-term mesophilic composting. Compost Science \& Utilization, 6:4, 67-73.

Mohee, R. and Mudhoo, A. (2005). Analysis of the physical properties of an in-vessel composting matrix. Powder Technology 155 (2005) 92-99. Available online at www.sciencedirect.com

Mondini, C.; Dell'Abate, M.T.; Leita, L. and Benedetti, A. 2003. An integrated chemical, thermal and microbilogical approach to compost stability evaluation. Journal of Environment Quality 32, 2379-2386.

Montgomery, D. y Runger, G. (1998). Probabilidad y Estadística aplicadas a la Ingeniería. Mc Graw Hill. 486 pp.
Shiklomanov, I. A. (1993). 'World water resources' in P. H. Gleick (ed) Water in Crisis,

Oxford University Press, New York \& Oxford.

Shyamala D.C. and Belagali, S. L. 2012. Studies on Variations in Physico-Chemical and Biological Characteristics at Different Maturity Stages of Municipal Solid Waste Compost. International Journal of Environmental Sciences Volume 2, N 4, 2012.

Termorshiuzen, A. J.; Moolenaar, S. W.; Veeken, A. H. M and Blok, W. J. 2004. The value of compost. Reviews in Environmental Science and Bio/Technology (2004) 3: 343-347. Springer 2005.

Tittarelli, F., Trinchera, A., Intrigliolo, F. and Benedetti, A. 2002. Evaluation of organic matter stability during the composting process of agroindustrial wastes. In: Imsam, H., Riddech, N., Klammer, S. (Eds), Microbilogy of Composting. Springer, Heidelberg, Germany, pp. 397-404. Thompson, W.; Leege, P.; Millner, P. and Watson, M. 2001. Test Methods for the Examination of Composting and Compost. Joint Project of The United States Department of Agriculture and The United States Composting Council. Zucconi, F.; De Bertoldi, M.; Ferranti, M.; L'Hermite, P. (1986). Compost: Production, Quality and Use. Elsevier Appl. Sci., NY. 853 pp. 